\title{
Improving Human Gait Recognition Using Feature Selection
}

\author{
Faezeh Tafazzoli ${ }^{1}$, George Bebis ${ }^{2}$, Sushil Louis ${ }^{2}$ and Muhammad Hussain ${ }^{3}$ \\ ${ }^{1}$ Department of computer Science and Engineering University of Louisville \\ faezeh.tafazzolialouisville.edu \\ ${ }^{2}$ Department of Computer Science and Engineering University of Nevada, Reno \\ \{bebis, sushil\}@cse.unr.edu \\ ${ }^{3}$ Department of Software Engineering, King Saud University, Riyadh, Saudi Arabia \\ mhussain@ksu.edu.sa
}

\begin{abstract}
Human gait, a biometric aimed to recognize individuals by the way they walk has recently come to play an increasingly important role in visual surveillance applications. Most of the existing approaches in this area, however, have mostly been evaluated without explicitly considering the most relevant gait features, which might have compromised the performance. In this paper, we have investigated the effect of discarding irrelevant or redundant gait features, by employing Genetic Algorithms (GAs) to select an optimal subset of features, on improving the performance of a gait recognition system. Experimental results on the CASIA dataset demonstrate that the proposed system achieves considerable gait recognition performance.
\end{abstract}

\section{Introduction}

Biometric-based identification of human using physiological or behavioral characteristics, due to their universality and uniqueness, are of great importance in surveillance and security applications. Many biometric-based authentication methods have been proposed using a wide variety of cues, such as fingerprint, iris, face, and gait. Among them, gait, has attracted considerable attention due to its ability to ascertain people's identity at a distance, with a low resolution image, while being noninvasive and non-perceivable [1]. However, human gait analysis involves challenging issues due to the highly flexible structure and self-occlusion of the human body. These issues mandate using complicated processes for the analysis of gait in markerless sequences [1].

A variety of gait recognition systems have been proposed in the literature. Typically, a large number of features are extracted to avoid loss of important gait information. Without employing some kind of feature selection strategy, however, many of the gait features being extracted could be redundant or irrelevant to the recognition task. In general, feature selection could provide valuable clues in terms of understanding the underlying distinctness among human gait patterns by reducing the number of features used in classification while maintaining acceptable classification accuracy. 
In particular, Genetic algorithms (GAs) offer a particularly attractive approach for this kind of problems since they are generally quite effective for rapid global search of large, non-linear and poorly understood spaces [2]. The use of feature selection, however, has not been given enough consideration in gait recognition. There are only some approaches [1, 3, 4 and 5], which have mainly considered conventional dimensionality reduction or statistical tools.

Motivated by our previous work on gender/face/vehicle classification using feature subset selection [6, 7], we propose using GAs on an improved silhouette based gait recognition approach to search the space of gait pattern feature vectors and demonstrate the importance of feature selection. Our experimental results show considerable Correct Classification (CCR) improvement compared to the results of KPCA feature vectors.

The rest of this paper is organized as follows: Section 2 presents a review of gait recognition approaches with emphasis on gait feature extraction. Section 3 overviews the proposed approach. Experimental results on different parameters of GA along with a comparison with a complete set of features are presented in Section 4. Finally, Section 5 concludes the paper and refers to future extensions of this research.

\section{Literature Review}

\subsection{Gait Recognition}

Gait recognition methods can be broadly divided into two categories: model-based and model-free. Model-based methods attempt to explicitly model the human body or motion by employing static and dynamic body parameters, which are typically view and scale invariant. Different approaches have used features like distances or angles between different human body parts $[1,8,9]$, trajectories of joint angles, head or feet $[10,11,12]$ using 2D stick models, motion parameters using 3D temporal models [13], or a combination of kinematics and appearances of a gait [14]. Model-free approaches, on the other hand, usually employ either shape of binary silhouettes or the whole motion of walking person's body, rather than modeling the whole human body or any part of it. These approaches are insensitive to the quality of silhouettes and have the advantage of low computational costs. However, they are usually not robust to viewpoint change and scale [6]. Some examples of this category are template matching of silhouettes [15], silhouette self-similarity [16], Gait Energy Image (GEI) [17], frame difference energy image (FDEI) [18], temporal patterns of gait [19] and adopting Gabor filters to decompose body shape into orientations and scales [20].

\subsection{Feature Selection}

Feature selection is essentially an optimization problem that involves searching the space of possible feature subsets to find one that is optimal or near optimal with respect to a certain criterion [6]. An exhaustive search is computationally prohibitive especially when there are a large number of features; this has led to the development of a wide range of feature selection methods [21, 22]. Generally, feature subset 
selection algorithms can be classified into two categories based on whether feature selection is performed independently of the learning algorithm used to construct the verifier or not. The former is computationally more efficient but its major drawback is that an optimal selection of features may not be independent of the inductive and representational biases of the learning algorithm that is used to build the classifier. On the other hand, the second type involves the computational overhead of evaluating a candidate feature subset by executing a selected learning algorithm on the database using each feature subset under consideration [2]. These weaknesses cause this kind of feature selection methods to get locked at a local minimum during the search process.

An alternative is the use of GAs, a form of inductive learning strategy, adaptive search techniques which have demonstrated substantial improvement over a variety of random and local search methods. This is accomplished by their ability to exploit accumulating information about an initially unknown search space in order to bias subsequent search into promising subspaces. The major reason for GA's popularity in various search and optimization problems is its global perspective, wide spread applicability and inherent parallelism. Various methods like [23, 24], have used GAs for their feature selection in the literature, based on the fact that usually GAs are more robust at the expense of more computational effort.

\section{Proposed Method}

The technique presented in this paper chooses the best feature vector with highest discrimination among a large number of features for every person extracted from his silhouette contour projections. This is accomplished through exploiting GA on eigenvectors of four contour projections of the silhouette of person accumulated over the whole sequence. The goal is encoding mostly important information about identity of the target concept of interest.

\subsection{Gait Pattern Extraction}

In the first phase a background subtraction technique is applied on each sequence. The silhouettes extracted in this process will be later used in the stage of gait pattern representation. The method used here for foreground segmentation is based on using the minimum graph cut method proposed in [25]. In this approach standard methods based upon graph flow will find an optimal cut in the graph built based upon the image, separating the foreground from the background. Typical result of applying foreground extraction is shown in Figure 1 for an input frame instance. In the next step, a velocity-filtering algorithm is employed to determine the bulk motion of the silhouette of subject. This algorithm will be applied on moving edges of each frame. These edges are extracted as the common output of background subtraction and edge detection. Using this motion information, a global temporal accumulation describing the person's average shape is formed over the gait sequence. Using a velocity-filtering 
algorithm [26], it is possible to determine object motion independent of shape, based on

$$
A_{v}(i, j)=\sum_{n=0} I_{n}[i+v \times n, j]
$$

where $A_{v}$ is the accumulation for velocity $v$ (in pixels per frame), $I_{n}$ is the image intensity function at frame $\mathrm{n}, \mathrm{i}$ and $\mathrm{j}$ are coordinate indices and $\mathrm{N}$ is the number of frames in the gait sequence. This algorithm sorts the objects in the scene according to their velocity and starting position, producing an accumulation for each possible object velocity. The highest peak in the plot of maximal intensity versus velocity indicates the object's velocity.

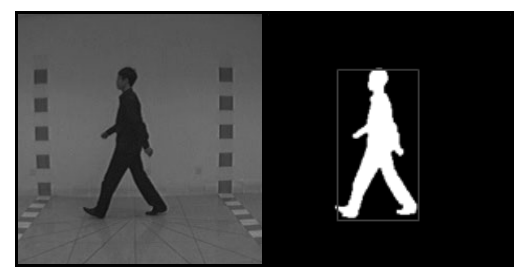

Fig. 1. Foreground segmentation applied on a sample sequence from the CASIA dataset

Having an approximate height and velocity for each subject, the last static parameter to be estimated is the period of walking. The motion of a person's limb during normal gait creates a complex periodic pattern, composed of many different components, which can be approximately modeled by a single sinusoid. The gait frequency and phase are particularly useful components since they describe this motion to a large extent and can be easily extracted without resolving limb dynamics, like variation of the width of silhouette.

\subsection{Gait Representation}

In this paper we have used an improved spatio-temporal gait representation as the basic gait pattern proposed in [27]. This pattern is obtained by using multi-projections of silhouette. Input silhouettes are first normalized based on the person's approximate height gained through global temporal accumulation of previous phase and then they are horizontally aligned. Gait pattern is created from the projections of silhouettes (Figure 2) which are generated from a sequence of binary silhouette images, $B_{t}(x, y)$, $\mathrm{B}_{\mathrm{b}}(\mathrm{x}, \mathrm{y}), \mathrm{B}_{\mathrm{r}}(\mathrm{x}, \mathrm{y})$ and $\mathrm{B}_{\mathrm{l}}(\mathrm{x}, \mathrm{y})$, indexed spatially by pixel location $(\mathrm{x}, \mathrm{y})$ and temporally by time $\mathrm{t}$. The distance vectors are the differences between the bounding box and the outer contour of silhouette on the related side. Each gait pattern, hence, is created as a new 2D image. For instance, gait pattern image for top-projection is formulated as $\mathrm{P}^{\mathrm{T}}(\mathrm{x}, \mathrm{t})=\sum_{\mathrm{y}} \mathrm{B}_{\mathrm{t}}(\mathrm{x}, \mathrm{y})$ where each column (indexed by time $\mathrm{t}$ ) is the top-projections (row sum) of silhouette image $\mathrm{B}_{\mathrm{t}}(\mathrm{x}, \mathrm{y})$. The result is a $2 \mathrm{D}$ pattern formed by stacking row projections together to form a spatio-temporal pattern. Other projections are constructed in the same way for bottom, left and right projections. Figure 3 shows an 
example of each pattern. It is clear that the distance vector is roughly periodic and gives the extent of movement of different parts of the subject. The brighter a pixel in $2 \mathrm{D}$ pattern in the following figure, the larger the value of the distance vector in that position.

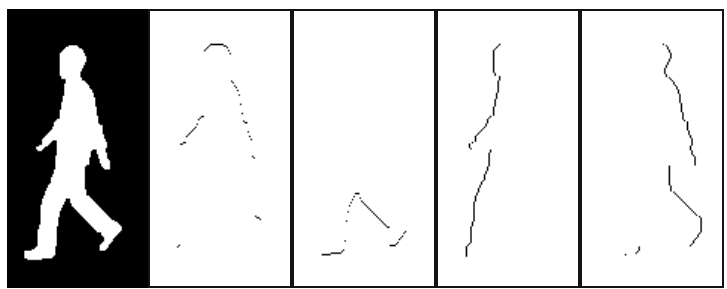

Fig. 2. A sample silhouette and the distance vectors corresponding to its four projections

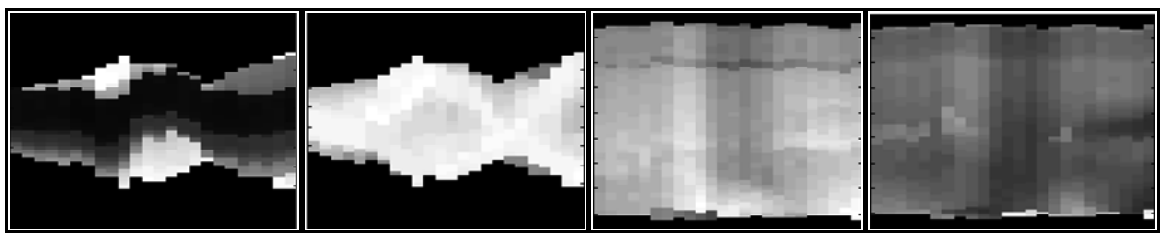

Fig. 3. From left to right: Spatio-temporal gait patterns estimated for top, bottom, left and right projections for a sample sequence

\subsection{Feature Extraction Using Kernel PCA}

In the next phase, we performed a dimensionality reduction procedure on the gait patterns extracted in previous step. As a result, training gait features that form feature database are obtained. Conventional linear subspace methods such as principal component analysis (PCA) can only produce linear subspace feature extractors. These are unsuitable for highly complex and nonlinear data distributions. In contrast, kernel subspace methods such as KPCA can capture higher order statistics present in a dataset, thus producing nonlinear subspaces for better feature extraction. In principle, kernel methods map the data to a higher dimensional feature space where conventional linear subspace methods can be used, with the resulting subspaces being nonlinear with regards to the original input space. Experiments and comparisons have shown that KPCA almost always outperforms PCA [28].

Usually we need to keep a smaller number of eigenvectors corresponding to the largest eigenvalues. It, however, has been found in several studies that different eigenvectors encode different kind of information [6]. In essence, different tasks make different demands in terms of the information that needs to be processed, and that this information is not contained in the same ranges of eigenvectors. Hence, we apply GA to search the space of eigenvectors with the goal of selecting a subset of them, which encode important information about the manner of walking for each individual. 


\subsection{Genetic Feature Subset Selection}

Evolutionary algorithms offer a particularly attractive approach to multi-criteria optimization because they are effective in high-dimensional search spaces. A GA is a model of machine learning that derives its behavior from a metaphor of some of the mechanisms of evolution in nature. This is done by the creation within a machine of a population of individuals represented by chromosomes. The individuals represent candidate solutions to the optimization problem being solved. It is assumed that the quality of each candidate solution can be evaluated using a fitness function. The main issues in applying GAs to any problem are selecting an appropriate representation and an adequate evaluation function. GAs use some form of fitness-dependent probabilistic selection of individuals from the current population to produce individuals for the next generation. The selected individuals are submitted to the action of genetic operators to obtain new individuals that constitute the next generation. The process of fitness-dependent selection and application of genetic operators to generate successive generations of individuals is repeated many times until a termination criterion is satisfied. In practice, the performance of GA depends on a number of that we have explored in section 4 .

Encoding. The simplest form of representation is binary representation. An individual of length 1 corresponds to a 1-dimensional binary feature vector, where each bit represents the elimination or inclusion of the associated feature.

Fitness Evaluation. Each subject is classified using the current feature subset. The overall fitness function will be evaluated by adding the weighted sum of the match score of that bit string indicating present features along with the number of features exploited meanwhile. However, the former part is the major concern. The final fitness function is defined as:

\section{Fitness $=100 \times$ Correct Recognition Rate $($ CRR) - Number of selected fea- tures/Chromosome length}

Initial Population. To generate the initial population we have created a random number for each chromosome to define the number of present features. These value, also are scattered randomly through the whole chromosome. So we would have a population with different permutations and number of features.

Selection. Elitism and linear ranking have been employed as selection techniques. Elitism reserves a slot in the next generation for the current highest scoring chromosome, without allowing that chromosome to be crossed over in the next generation. This method can very rapidly increase performance of GA, by avoiding loss of the best found solution. In ranking selection each individual in the population is assigned a numerical rank based on fitness and selection is based on these rankings rather than absolute differences in fitness. The advantage of this method is that it can prevent very fit individuals from gaining dominance early at the expense of less fit ones. 
Operators. To explore the whole space of features crossover and mutation operators must be employed. In the proposed method a uniform crossover is exploited to avoid destroying the schema in case of presence of dependency among neighbor eigenvectors. Mutation also, is applied using the bitwise method.

Finally, three different classifiers, K-Nearest Neighbor and Naive Bayes Classifiers and SVM, have been utilized to evaluate chromosomes during fitness estimation.

\section{Experimental Results}

The proposed approach has been tested on CAIA database [29] which consists of 268 sequences from 124 subjects. The sequences were collected many viewing angles ranging from 0 to 180 incrementing 18 degrees. In our experiments the fronto-parallel sequences have been utilized. For each subject, there are 6 trials. Data collection is done indoors with simple background. All the video sequences were stored as video files encoded with mjpeg codec. The frame size of the video files was $320 \times 240$, with frame rate of $25 \mathrm{fps}$.

To extract the features and applying KPCA on temporal contour projection images, a fixed number of frames should be selected from each sequence to have chromosomes with same length eventually. This value was determined based on the velocity and period of walking for each individual so that at least the selected frames would include one cycle of walking; the frames also were chosen based on the starting phase of each person. KPCA was then applied on the temporal contour projections of these frames using Gaussian function as the kernel. A normalized bounding box of $141 \times 56$ with 25 frames was considered. This process led in to $56 \times 25$ eigenvectors for top and bottom gait patterns, and $141 \times 25$ eigenvectors for left and right projections, resulting in 9850 eigenvectors totally. These features were presented to a GA with population size of 800, 1000 and 1500, during 80, 200 and 100 generations, to observe the effect of various parameters of GA on algorithm performance. As mentioned earlier, each subject has 6 sequences, 3 of which were selected randomly as test set. Among the rest of streams, two were employed for training and the remaining one for validation. This process was repeated 7 times. The final CRR is the average value of these iterations.

Another important parameter is the size of population; since the length of each chromosome is too long, number of population should be large enough to be able to contain various types of feature permutations in each generation. The number of generations, on the other hand, should be large enough to let better chromosomes overcome the population gradually. The other parameter is the type of classifier which affects the overall fitness directly. Three classifiers have been employed. Since the evaluation value of each chromosome is determined mainly from the accuracy of classifier, different techniques result in different outputs of features.

Table 1 shows our results before applying GA and Table 2 summarizes Recognition rates after feature subset selection with GA using different parameters. According to these figures and tables, it is observable that GA in all cases has decreased the number of eigenvectors used in classification while improving Correct Classification 
Rate (CCR) considerably. In experiment 1 , the results of which are displayed in the first column of Table 1, all features have been exploited in classification. In experiment 2 , on the other hand, just $10 \%$ of eigenvectors with higher eigenvalues have been selected as final features, which are supposed to have good discriminatory ability based on the main concept of KPCA (second column). There is however, considerable improvement in CCR values in comparison with values of 6th column of Table 2 exhibiting the effect of GA. These experiments also demonstrate that eigenvectors encoding irrelevant or redundant information have not been favored by the GA approach.

Table 1. Averaged performance in all experiments before GA

\begin{tabular}{cc}
\hline CCR using all features & CCR using top 10\% eigenvectors \\
\hline $77.7 \%$ & $77.8 \%$
\end{tabular}

Table 2. Overall performance after GA

\begin{tabular}{ccccccc}
\hline Pop & Classifier & Selection & Crossover P. & Mutation P. & CCR & Selected Features \\
\hline 800 & Naïve Bayes & Elitism & 0.6 & 0.1 & $65.7 \%$ & $14.7 \%$ \\
800 & KNN & Elitism & 0.6 & 0.1 & $63.2 \%$ & $28.5 \%$ \\
800 & SVM & Elitism & 0.6 & 0.1 & $73.6 \%$ & $32.5 \%$ \\
1000 & Naïve Bayes & Elitism & 0.9 & 0.05 & $79.3 \%$ & $14.4 \%$ \\
1000 & Naïve Bayes & Elitism & 0.6 & 0.1 & $84.9 \%$ & $19.8 \%$ \\
1500 & KNN & Elitism & 0.6 & 0.1 & $86.3 \%$ & $31.8 \%$ \\
1500 & KNN & Ranking & 0.6 & 0.1 & $81.8 \%$ & $26.4 \%$ \\
1500 & SVM & Elitism & 0.6 & 0.1 & $\mathbf{8 8 . 6 \%}$ & $44.1 \%$ \\
\hline
\end{tabular}

We have performed a comparison based on the selected features (Table 3). In this experiment we chose GEI features which have proven to achieve good results in case of gait recognition. GEI represents gait using a single image which contains information about both body shape and human walking dynamics. GEI is thus a compact representation which makes it an ideal starting point for feature selection since it is computational expensive if the number of features to select is high [17]. Table 4 demonstrates the results of recognition using features extracted by KPCA from GEI representations. We can see that their performance has not been as good as the results of Table 1. We should mention that in this experiment only the normal sequences with view point of 90 degrees have been employed to keep the consistency with our previous experiments. One of the reasons that GEI did not perform well in the second dataset is that although it is comparatively robust to noise, it loses the dynamical variation between successive frames which was emphasized on in case of contour projections. To check the effect of feature selection on a different type of feature we applied GA on this data. As we can see, the accuracy has increased considerably using appropriate features from selected eigenvectors of GEI images. 
Table 3. Recognition results using GEI features

\begin{tabular}{ccc}
\hline CCR using all features & CCR using top 10\% eigenvectors & $\begin{array}{c}\text { CCR using feature subset } \\
\text { selection from GA }\end{array}$ \\
\hline
\end{tabular}

$58.8 \%$

$46.6 \%$

$82.9 \%$

\section{Conclusion}

We have described a method for extracting the gait signatures and kinematic features for analyzing and identifying the gait motion. Temporal contour projections have been employed as gait patterns from which we were able to extract required feature vectors for classification.

First, some static parameters were estimated; these values were later used for data normalization from which gait patterns were extracted. Eigenvectors calculated from applying KPCA on gait patterns yielded a large number of features that can be reduced with GA. The classification results demonstrated the power of GA in selecting the best subset of feature which led to a promising recognition rate compared to the whole feature vector which we got after KPCA. In our future work, we will generalize the method to other imaging viewpoints and outdoor data, and further analyze the above features for constructing a method capable of operating in real-world applications.

\section{References}

1. Yoo, J., Nixon, M.S.: Automated Marker-less Analysis of Human Gait Motion for Recognition and Classification. ETRI Journal 33(2), 259-266 (2011)

2. Oliveir, L.S., Benahmed, N., Sabourin, R., Bortolozzi, F., Suen, C.Y.: Feature subset selection using genetic algorithms for handwritten digit recognition. In: XIV Brazilian Symposium on Computer Graphics and Image Processing, Florianopolis, Brazil, pp. 362-369 (October 2001)

3. Han, J., Bhanu, B.: Individual recognition using gait energy image. IEEE Transactions on Pattern Analysis and Machine Intelligence 28(2), 316-322 (2006)

4. Veres, G., Gordon, L., Carter, J.N., Nixon, M.: What image information is important in silhouette-based gait recognition? In: Proceedings of the 2004 IEEE Computer Society Conference on Computer Vision and Pattern Recognition, vol. 2, pp. 776-782 (July 2004)

5. Begg, K.: A machine learning approach for automated recognition of movement patterns using basic, kinetic and kinematic gait data. Journal of Biomechanics 38, 401-408 (2005)

6. Sun, Z., Bebis, G., Miller, R.: On-Road Vehicle Detection Using Evolutionary Gabor Filter Optimization. IEEE Transactions on Intelligent Transportation Systems 6(2), 125-137 (2005)

7. Sun, Z., Bebis, G., Yuan, X., Louis, S.J.: Genetic Feature Subset Selection for Gender Classification: A Comparison Study. In: Sixth IEEE Workshop on Applications of Computer Vision (WACV 2002), pp. 165-170 (2002)

8. Yoo, J., Hwang, D., Moon, K., Nixon, M.S.: Automated Human Recognition by Gait using Neural Network. In: First Workshops on Image Processing Theory, Tools and Applications (IPTA), pp. 1-6 (November 2008) 
9. Bobick, A.F., Johnson, A.Y.: Gait recognition using static activity-specific parameters. In: Proceedings of IEEE Computer Society Conference on Computer Vision and Pattern Recognition (CVPR 2001), Kauai, Hawaii, pp. 423-430 ( December 2001)

10. Tanawongsuwan, R., Bobick, A.F.: Gait recognition from time-normalized joint-angle trajectories in the walking plane. In: IEEE Computer Vision and Pattern Recognition Conference (CVPR 2001), Kauai, Hawaii, pp. 726-731 (December 2001)

11. Wang, A.H., Liu, J.W.: A gait recognition method based on positioning human body joints. In: International Conference on Wavelet Analysis and Pattern Recognition (ICWAPR 2007), Beijing, pp. 1067-1071 (November 2007)

12. Jean, F., Albu, A.B., Bergevin, R.: Towards view-invariant gait modeling: Computing view-normalized body part trajectories. Pattern Recognition 42(11), 2936-2949 (2009)

13. Urtasun, R., Fua, P.: 3D Tracking for Gait Characterization and Recognition. In: Sixth IEEE International Conference on Automatic Face and Gesture Recognition, pp. 17-22 (May 2004)

14. Zhang, X., Fan, G.: Dual gait generative models for human motion estimation from a single camera. IEEE Transactions on System, Man and Cybernetics, Part B: Cybernetics 40(4), 1034-1049 (2010)

15. Collins, R.T., Gross, R., Shi, J.: Silhouette-based human identification from body shape and gait. In: Fifth IEEE International Conference on Automatic Face and Gesture Recognition, Washington, DC, USA, pp. 366-371 (May 2002)

16. BenAbdelkader, C., Cutler, R., Davis, L.: Gait recognition using image self-similarity. EURASIP Journal on Applied Signal Processing 2004(4), 572-585 (2004)

17. Zheng, S., Zhang, J., Huang, K., He, R., Tan, T.: Robust View Transformation Model for Gait Recognition. In: International Conference on Image Processing (ICIP), Brussels, Belgium (2011)

18. Chen, C., Liang, J., Zhao, H., Hu, H., Tian, J.: Frame difference energy image for gait recognition with incomplete silhouettes. Pattern Recognition Letters 30(11), 977-984 (2009)

19. Dadashi, F., Araabi, B.N., Soltanian-Zadeh, H.: Gait Recognition Using Wavelet Packet Silhouette Representation and Transductive Support Vector Machines. In: 2nd International Congress on Image and Signal Processing, Tianjin, pp. 1-5 (October 2009)

20. Hu, M., Wang, Y., Zhang, Z., Wang, Y.: Combining spatial and temporal information for gait based gender classification. In: 20th International Conference on Pattern Recognition (ICPR), pp. 3679-3682 (August 2010)

21. Ephzibah, E.P.: Cost Effective Approach on Feature Selection using Genetic and Fuzzy Logic for Diabetes Diagnosis. International Journal on Soft Computing (IJSC) 2(1), 1-10 (2011)

22. Dash, M., Liu, H., Motoda, H.: Consistency Based Feature Selection. In: Terano, T., Liu, H., Chen, A.L.P. (eds.) PAKDD 2000. LNCS, vol. 1805, pp. 98-109. Springer, Heidelberg (2000)

23. Yang, J., Honavar, V.: Feature subset selection using a genetic algorithm. In: Motoda, H., Liu, H. (eds.) A Data Mining Perspective, ch. 8. Kluwer, Dordrecht (1998)

24. Chtioui, Y., Bertrand, D., Barba, D.: Feature selection by a genetic algorithm, application to seed discrimination by artificial vision. J. Sci. Food Agric. 76, 77-86 (1998)

25. Howe, N.R., Deschamps, A.: Better foreground segmentation through graph cuts. Computer Vision and Pattern Recognition 94(11), 49-52 (2007)

26. Wagg, D.K., Nixon, M.S.: Model-based gait enrolment in real-world imagery. In: Workshop on Multimodal User Authentication, Santa Barbara, CA, USA, pp. 11-12 (December 2003) 
27. Ekinci, M., Aykut, M.: Human Gait Recognition based on Kernel PCA Using Projections. Journal of Computer Science and Technology 22(6), 867-876 (2007)

28. Chin, T.J., Suter, D.: Incremental Kernel Principal Component Analysis. IEEE Transactions on Image Processing 16(6), 1662-1674 (2007)

29. Yu, S., Tan, D., Tan, T.: A Framework for Evaluating the Effect of View Angle, Clothing and Carrying Condition on Gait Recognition. In: Proceeding of the 18'th International Conference on Pattern Recognition (ICPR), Hong Kong, China (August 2006) 